\title{
L’avenir des groupes linguistiques au Québec : équilibres et options
}

\section{THE FUTURE OF LINGUISTIC GROUPS IN QUEBEC: THE IMPACT OF IMMIGRATION \\ EL PORVENIR DE LOS GRUPOS LINGUISTICOS EN QUEBEC : EQUILIBRIOS Y OPCIONES}

\author{
Norbert Robitaille, Robert Bourbeau, Chantal Girard et Marc Tremblay
}

Volume 21, numéro 2, automne 1992

Montréal, $\mathrm{XIX}^{\mathrm{e}}-\mathrm{XX}^{\mathrm{e}}$ siècles : croissance urbaine et diversité culturelle

URI : https://id.erudit.org/iderudit/010125ar

DOI : https://doi.org/10.7202/010125ar

Aller au sommaire du numéro

Éditeur(s)

Association des démographes du Québec

ISSN

0380-1721 (imprimé)

1705-1495 (numérique)

Découvrir la revue

Citer cet article

Robitaille, N., Bourbeau, R., Girard, C. \& Tremblay, M. (1992). L'avenir des groupes linguistiques au Québec : équilibres et options. Cahiers québécois de démographie, 21(2), 147-161. https://doi.org/10.7202/010125ar
Résumé de l'article

Durant la période 1981-1986, l'équilibre des phénomènes démographiques au Québec était tel que la proportion de francophones avait tendance à s'accroître. Cet article projette cette situation ainsi que d'autres scénarios, et montre l'impact d'un accroissement de l'immigration sur le pourcentage de francophones au Québec. Il souligne, pour le Québec, l'antinomie qui existe entre la croissance de l'effectif de la population dans son ensemble et celle du pourcentage de francophones. Plus spécifiquement, il montre que la politique du gouvernement qui prévoit une immigration de 55000 immigrants annuellement entraînerait selon toute vraisemblance une diminution du pourcentage de francophones.
Tous droits réservés @ Association des démographes du Québec, 1992

Ce document est protégé par la loi sur le droit d'auteur. L'utilisation des services d'Érudit (y compris la reproduction) est assujettie à sa politique d'utilisation que vous pouvez consulter en ligne.

https://apropos.erudit.org/fr/usagers/politique-dutilisation/ 
Cahiers québécois de démographie

Vol. 21, no 2, automne 1992, p. 147-161.

\title{
L'avenir des groupes linguistiques au guébec : équilibres et options
}

\author{
Norbert ROBITAILLE, Robert BOURBEAU, \\ Chantal GIRARD et Marc TREMBLAY *
}

L'analyse démographique des groupes linguistiques québécois a tout d'abord traité de l'avenir prévisible des francophones (Charbonneau, Henripin et Légaré, 1969; Charbonneau et Maheu, 1973) puis, plus récemment, des anglophones (Lachapelle et Henripin, 1980; Henripin, 1984; Caldwell, 1984). En fait, à la fin des années soixante et au début des années soixante-dix, on s'inquiêtait de la baisse de la proportion des francophones (langue maternelle) au sein de la population du Québec : de 1951 à 1971 , cette proportion est passée de $82,5 \%$ à $80,7 \%$. Il y eut rupture d'un certain équilibre linguistique en vertu duquel la proportion de francophones s'accroissait légèrement ou du moins se maintenait au fil des recensements depuis 1931 (tableau 1). Les causes principales de cette rupture d'équilibre sont bien connues; ce sont l'augmentation de l'immigration internationale au cours des années cinquante et la disparition progressive de la surfécondité des francophones au cours des années soixante.

Durant la deuxième moitié des années soixante-dix et au début des années quatre-vingt, on s'est plutôt inquiété du sort de la minorité anglophone : non seulement son érosion en proportion de la population totale se poursuivait-elle, mais on

* Norbert Robitaille et Robert Bourbeau : Groupe de recherche sur la démographie québécoise et Département de démographie de l'Université de Montréal; Chantal Girard : Conseil régional de la santé et des services sociaux de l'Abitibi-Témiscamingue; Marc Tremblay : Université du Québec à Chicoutimi, Centre interuniversitaire de recherches sur les populations (SOREP). Les travaux qui ont conduit à cet article ont été réalisés grâce à l'assistance financière du Conseil de recherches en sciences humaines du Canada (CRSHC, subvention 410-88-1149). 
TABLEAU 1 - Répartition de la population du Québec selon la langue maternelle (\%) 1931-1986 et selon la langue d'usage, 1971-1986

\begin{tabular}{|c|c|c|c|c|c|c|}
\hline & \multicolumn{3}{|c|}{ Langue maternelle } & \multicolumn{3}{|c|}{ Langue d'usage } \\
\hline & Français & Anglais & Autre & Français & Anglais & Autre \\
\hline 1931 & 79,7 & 15,0 & 5,3 & & & \\
\hline 1941 & 81,5 & 14,1 & 4,4 & & & \\
\hline 1951 & 82,5 & 13,8 & 3,7 & & & \\
\hline 1961 & 81,2 & 13,3 & 5,6 & & & \\
\hline 1971 & 80.7 & 13,1 & 6.2 & 80.8 & 14.7 & 4,5 \\
\hline 1976 & 81,1 & 12,8 & 6,1 & & & \\
\hline 1981 & 82,4 & 11,0 & 6,6 & 82,8 & 12.3 & 4.9 \\
\hline $1986^{a}$ & 82,9 & 10,3 & 6,8 & 83,1 & 11,8 & 5,1 \\
\hline
\end{tabular}

Source : Statistique Canada, Recensements du Canada, 1931 à 1986.

a. Les réponses multiples ont été réparties dans les mèmes proportions que celles du recensement de 1981 .

constatait une baisse de son effectif total à partir de 1976. La très forte augmentation d'une propension déjà élevée des anglophones à quitter le Québec expliquait en bonne partie ce phénomène (Bourbeau, 1986).

Ainsi, toute rupture significative d'un équilibre linguistique reconnu tacitement suscite des interrogations. Le plus souvent, le réflexe premier des démographes est d'utiliser des modèles de projections pour appréhender les effets possibles des modifications observées dans les phẻnomènes démolinguistiques sur la composition linguistique à moyen et à long terme. Les derniers travaux de ce genre pour l'ensemble du Québec remontent à un certain temps et étaient basés sur la connaissance des effectifs recensés, soit en 1971 (Lachapelle et Henripin, 1980), soit en 1976 (Robitaille et Bourbeau, 1982), ou au mieux en 1981 (Termote et Gauvreau, 1988). Aucune étude ${ }^{1}$ n'a pris en compte les données du recensement de 1986, ni pour projeter les effectifs de la population selon la langue maternelle ni pour évaluer le bilan démolinguistique de la période 1981-1986.

1 Cet article a été soumis en avril 1992. Depuis, certaines études ont paru, par exemple la contribution de Marc Termote à un document publié par le Conseil de la langue française en 1993 (Marc V. Levine et al., Contextes de la politique linguistique québécoise. Analyse juridique, démographique, économique et culturelle présentée au séminaire du Conseil de la langue française, du 12 au 14 novembre 1992, Québec, Les Publications du Québec, 121 pages); voir aussí, du mème auteur : "Langue : quand les prévisions démographiques dérangent, La Presse, 23 juin 1993 : B2. 
Dans le cadre de nos dernières recherches, nous avons tiré parti des informations les plus récentes sur le renouvellement des groupes linguistiques québécois pour présenter une perspective d'analyse un peu différente des travaux classiques de perspectives démolinguistiques. Plutôt que de nous interroger sur l'avenir le plus probable du groupe francophone, nous nous sommes d'abord demandé quels sont les phénomènes les plus susceptibles d'entraîner une modification (rupture d'équilibre) de l'importance relative des francophones au Québec. Puis nous avons exploré quelques combinaisons de phénomènes susceptibles d'assurer le maintien du pourcentage de ces derniers.

Comme les populations humaines en général, les groupes linguistiques se renouvellent par l'accroissement naturel (féconditê et mortalitê) et la migration. Cependant vu la possibilité de changer de langue, on doit ajouter à ces phênomènes classiques la mobilité linguistique. Cet article reprend en les synthétisant et en les adaptant la problématique et les résultats du mémoire de Chantal Girard (1992). Celle-ci a mis au point un modèle de projection des groupes linguistiques qu'elle a utilisé pour analyser la sensibilité du pourcentage des francophones aux variations des différents phénomènes dans l'horizon 1981-2031.

Avant d'analyser les modifications des phénomènes, nous avons examiné la période de base 1981-1986 et l'avons projetée sur 35 ans (horizon 1981-2016). Ensuite, nous avons fait varier chacun des phénomènes composant cet équilibre afin d'en voir la conséquence pour l'importance des francophones. Afin de faciliter la comparaison de l'effet des phénomènes, nous nous sommes demandé quelle serait la variation nécessaire de chaque phénomène pour entraîner une diminution de $1 \%$ des francophones, leur maintien, ou une augmentation de $1 \%$, et ce, en gardant constants les autres phénomènes à leur niveau de 1981-1986, dernière période pour laquelle toute l'information était disponible, puisque le recensement de 1991 gardait encore ses secrets au moment où ces lignes ont été écrites.

L'horizon de 35 ans est un peu arbitraire, mais l'ordre de grandeur se justifie par le fait qu'il permet aux phénomènes lents de faire sentir leurs effets sans que l'exercice relève de la pure science fiction comme pour des horizons beaucoup plus longs (100 ans par exemple). 


\section{LE MODÈLE}

Le modèle de projection fonctionne de façon classique, selon la méthode des composantes. Il permet la projection, par groupe d'âge quinquennal, de quatre groupes linguistiques suivant la langue maternelle et la langue d'usage ${ }^{2}$. Rappelons que les modèles utilisés jusqu'à présent ne retenaient au maximum que trois groupes linguistiques, le français, l'anglais et le groupe résiduel. L'ajout d'un quatrième groupe permet la projection de n'importe quel groupe linguistique, si on dispose des hypothèses nécessaires. Dans le mémoire de Chantal Girard, le groupe italophone a été projeté. Cependant dans cet article, ce groupe sera parfois fondu dans le groupe résiduel (allophones), notre attention étant orientée vers le groupe francophone.

La mortalitê varie selon la langue d'usage comme le montre le tableau 2 pour la période 1981-1986. Dans l'analyse de sensibilité qui suivra, la mortalité a été maintenue à son niveau de 1981-1986. Nous nous fondons pour ce faire sur la vêrification faite par Chantal Girard dans son mémoire : "la proportion de francophones dans la population est peu sensible aux variations de la mortalité" (Girard, 1992).

La fécondité varie également selon la langue d'usage comme le montre le tableau 3. Dans les études de sensibilité, nous avons affecté tous les taux de fécondité par groupe d'âge d'un même facteur multiplicatif. Nous sommes conscients que cela introduit une légère distorsion puisque tous les groupes d'âge ne sont pas également susceptibles de modifier l'intensité de leur féconditê; nous avons cependant jugé cette erreur négligeable à l'échelle de l'impact des variations de l'intensité de la fécondité. Précisons que dans notre modèle, la langue maternelle des enfants a été obtenue à partir de la langue d'usage des mères.

La migration constitue un module plus complexe puisqu'il est nécessaire de ventiler les flux selon la langue maternelle et la langue d'usage, tant pour les immigrants que pour les émigrants. De plus, on peut distinguer les migrants selon que le lieu d'origine ou de destination se situe à l'intérieur du Canada (migrations interprovinciales) ou à l'extérieur (migrations internationales). Nous n'avons pas retenu cette distinction dans le cadre de ce premier article quoique le modèle nous le permette. Nous avons simplement considéré les migrants globalement, d'où qu'ils viennent et où qu'ils aillent.

2 Langue maternelle : première langue apprise et encore comprise. Langue d'usage : langue parlée habituellement (le plus souvent) à la maison. 
TABLEAU 2 - Espérance de vie à la naissance selon le groupe de langue d'usage, Québec, 1981-1986

\begin{tabular}{lcccl}
\hline & \multicolumn{4}{c}{ Langue d'usage } \\
\cline { 2 - 5 } Sexe & Français & Anglais & Italien & Autre \\
\hline Féminin & 78,53 & 80,77 & 82,71 & 82,71 \\
Masculin & 70,62 & 75,25 & 77,74 & 77,74 \\
\hline
\end{tabular}

Source : Girard, 1992.

TABLEAU 3 - Indice synthétique de fécondité a selon le groupe de langue d'usage. Québec 1981-1986

\begin{tabular}{lccccc} 
& \multicolumn{4}{c}{ Langue d'usage } & \\
& Français & Anglais & Italien & Autre & Total \\
\hline $\begin{array}{l}\text { Nombre moyen } \\
\text { d'enfants par } \\
\text { femme }\end{array}$ & 1,52 & 1.38 & 1,01 & 1.89 & 1,51 \\
\hline
\end{tabular}

Source : Girard, 1992.

a. L'indice synthétique de fécondité est le nombre d'enfants qu'aurait une femme, si elle était soumise à chaque àge de sa vie féconde à la féconditê du moment.

TABLEAU 4 - Immigrants (internationaux et interprovinciaux) selon la langue maternelle et la langue d'usage, Québec, 1981-1986

\begin{tabular}{lrrrrrr}
\hline & \multicolumn{5}{c}{ Langue d'usage } \\
\cline { 2 - 7 } mangue & \multicolumn{7}{c}{ maternelle } & Français & Anglais & Italien & Autre & Total \\
\hline Français & 46207 & 5831 & 5 & 3065 & 55108 \\
Anglais & 3398 & 35786 & 15 & 2040 & 41239 \\
Italien & 148 & 433 & 978 & 0 & 1559 \\
Autre & 4489 & 5775 & 0 & 31052 & 41316 \\
Total & 54242 & 47825 & 998 & 36 & 157 & 139222 \\
\hline
\end{tabular}

Source : Girard, 1992.

En ce qui concerne les immigrants, l'hypothèse se fera sur les nombres absolus à partir des estimations de la période 1981-1986 présentées au tableau 4. Ces immigrants seront répartis selon l'âge et le sexe d'après les structures observées en 1981-1986.

Nous avons recouru à deux types d'analyse de sensibilité en ce qui concerne l'immigration. Une première analyse consiste à faire varier le niveau total de l'immigration en multipliant l'effectif des immigrants selon la langue maternelle et d'usage 
TABLEAU 5 - Émigrants et sortants selon la langue maternelle et la langue d'usage, Québec, 1981-1986

\begin{tabular}{lrrrrrr}
\hline & \multicolumn{5}{c}{ Langue d'usage } \\
\cline { 2 - 7 } maternelle & Français & Anglais & Italien & Autre & Total \\
\hline Français & 43320 & 14647 & 0 & 271 & 58239 \\
Anglais & 2853 & 85925 & 0 & 775 & 89553 \\
Italien & 55 & 975 & 410 & 0 & 1440 \\
Autre & 589 & 8125 & 0 & 7221 & 15935 \\
Total & 46817 & 109673 & 410 & 8267 & 165168 \\
\hline
\end{tabular}

Source : Girard, 1992.

par un même facteur. Pour la seconde analyse, nous avons fait varier le pourcentage des francophones parmi les immigrants. Les immigrants qui sont retranchés du groupe de langue maternelle et d'usage autre sont ajoutés au groupe de langue maternelle et d'usage français.

Pour ce qui est de l'émigration, la situation 1981-1986 a été décomposée en deux étapes. Dans un premier temps, on applique à la population de chaque langue d'usage, par groupe quinquennal d'âge, sexes réunis, des probabilités d'émigrer dont on peut faire varier l'intensité. Dans un second temps, on multiplie le nombre d'émigrants par langue d'usage obtenus dans le premier temps par un facteur qui ventile ces émigrants suivant la langue d'usage et la langue maternelle. La migration générée pour la période 1981-1986 se retrouve dans le tableau 5 .

Le dernier phénomène à considërer concerne les transferts linguistiques. On sait que les individus sont susceptibles de changer de langue d'usage et, ce faisant, de changer de groupe linguistique. En dépit de difficultés inhérentes aux donnêes ${ }^{3}$, nous avons estimé la mobilitê linguistique pour la période 1981-1986. Nous avons supposé que ce phénomène se produisait avant l'âge de 40 ans et qu'il était irréversible et non renouvelable. Le modèle fonctionne de la façon suivante. Dans un premier temps, une série de probabilités types est appliquée à la population distribuée suivant l'âge et la langue maternelle. Il en découle un nombre de personnes qui effectuent un transfert

3 Les réponses multiples ont èté résolues de manière à rendre les données de 1986 comparables à celles de 1981; il est possible que cette façon de faire entraine la création de transferts fictifs et l'annulation de transferts réels. Cependant, étant donné l'importance de ce phénomène par rapport aux autres, nous ne pensons pas que le biais soit significatif. 
TABLEAU 6 - Transferts linguistiques, Québec, 1981-1986

\begin{tabular}{|c|c|c|c|c|c|}
\hline \multirow{2}{*}{$\begin{array}{l}\text { Langue } \\
\text { maternelle }\end{array}$} & \multicolumn{5}{|c|}{ Langue d'usage } \\
\hline & Français & Anglais & Italien & Autre & Total \\
\hline Français & -13442 & 13442 & 0 & 0 & 0 \\
\hline Anglais & 9964 & -9964 & 0 & 0 & 0 \\
\hline Italien & 865 & 1755 & -2620 & 0 & 0 \\
\hline Autre & 2529 & 5134 & 0 & -7663 & 0 \\
\hline $\begin{array}{l}\text { Transferts } \\
\text { nets }\end{array}$ & -84 & 10367 & -2620 & -7663 & 0 \\
\hline
\end{tabular}

Source : Girard, 1992.

Note : On remarquera l'absence de transferts vers l'italien et les autres langues qui est justifiée par le fait que nos données sont le fruit d'estimations et que la précision de celles-ci ne peut prétendre évaluer des nombres très petits qui seraient entachés d'une trop grande erreur.

linguistique. Dans un second temps, on applique un facteur de répartition au nombre de personnes qui ont subi un transfert de façon à les répartir dans les populations par langue d'usage. Le tableau 6 présente la direction des transferts linguistiques estimés à partir du recensement de 1986 pour la période 1981-1986.

On enregistre un peu plus de trente-trois mille transferts bruts (il s'agit de la somme des cellules du tableau 6 à l'exclusion de la diagonale). Ceux-ci favorisent les anglophones aux dépens des allophones, le phénomène étant à peu près neutre pour les francophones, dont les gains compensent à peu près les pertes.

Dans notre analyse de sensibilitê, nous n'avons pas fait varier l'intensité de la mobilité linguistique, mais plutôt le pourcentage des transferts effectués par les allophones qui profitent aux groupes anglophone $(67 \%)$ ou francophone $(33 \%)$ (estimation de la situation 1981-1986 à partir des données du recensement de 1986). On pourrait également faire varier l'intensité de la mobilité linguistique, mais il s'agit là, à notre avis, d'un aspect de la mobilité linguistique qui a beaucoup plus d'inertie que le sens de la mobilité linguistique.

\section{LES RESULTATS}

La figure 1 offre une synthèse de l'analyse de la sensibilité du pourcentage des francophones (langue d'usage) à des modifications de certains aspects des phénomènes régissant leur renouvellement. La présentation des résultats vise à montrer 


\begin{tabular}{|c|c|c|c|c|c|c|c|c|}
\hline \multicolumn{9}{|c|}{ Hypothèses de variation d'un seul phénomène à la fois } \\
\hline \multicolumn{4}{|l|}{ Immigr. (000) } & 216 & 188 & 161 & 139 & \\
\hline \multicolumn{4}{|l|}{$\%$ immigr. franc. } & 21 & 27 & 34 & 39 & \\
\hline \multicolumn{4}{|l|}{ Émigr. $(000)$} & 90 & 114 & 141 & 165 & \\
\hline \multicolumn{5}{|l|}{$\%$ vers français } & & 0 & 33 & 100 \\
\hline \multicolumn{5}{|l|}{ ISF } & & 1 & \multicolumn{2}{|c|}{$1,5 \mathbb{1}, 8$} \\
\hline \multicolumn{7}{|c|}{ Hypothèse de variation de deux phénomènes à la fois } & & \\
\hline \multicolumn{3}{|l|}{$\begin{array}{l}\text { Immigr. }(000) \\
\text { Êmigr. }(000)\end{array}$} & \multicolumn{2}{|c|}{$\begin{array}{l}216 \\
141\end{array}$} & & & & \\
\hline \multicolumn{7}{|c|}{ Hypothèses de variation de trois phénomènes à la fois } & & \\
\hline \multicolumn{4}{|c|}{$\begin{array}{lrr}\text { Immigr. (000) } & 216 & 216 \\
\% \text { immigr. franc. } & 27 & 34 \\
\text { Êmigr. (000) } & 141 & 141\end{array}$} & & & & & \\
\hline \multicolumn{7}{|c|}{ Hypothèses de variation de quatre phènomènes à la fois } & & \\
\hline $\begin{array}{l}\text { Immigr. (OOO) } \\
\% \text { immigr. franc. } \\
\text { Êmigr. (OOO) } \\
\% \text { vers français }\end{array}$ & \multicolumn{3}{|c|}{$\begin{array}{rr}216 & 216 \\
30 & 30 \\
141 & 141 \\
& 100\end{array}$} & & & & & \\
\hline \multirow[t]{2}{*}{$\begin{array}{l}\text { Pourcentage des } \\
\text { francophones } 77,5\end{array}$} & 78,5 & 79,5 & 80,5 & 81,5 & 82,5 & 83,5 & 84,5 & \\
\hline & $\begin{array}{c}1 \\
-0,04\end{array}$ & $\begin{array}{c}1 \\
-0,03\end{array}$ & $\begin{array}{c}1 \\
-0,02\end{array}$ & $\begin{array}{c}1 \\
-0,01\end{array}$ & $\begin{array}{l}1 \\
0\end{array}$ & $\begin{array}{c}1 \\
0,01\end{array}$ & $\begin{array}{c}1 \\
0,02\end{array}$ & $\begin{array}{c}1 \\
0,03\end{array}$ \\
\hline
\end{tabular}

Variation du pourcentage des francophones après 35 ans

NOTE EXPLICATIVE - Le pourcentage des francophones qui est donné en bas de la figure indique le pourcentage que représenteraient les francophones du guébec si les hypothèses mentionnées au-dessus étaient maintenues constantes durant 35 ans. Lorsqu'une seule hypothèse se retrouve entre deux lignes horizontales, cela signifie que les autres hypothèses sont maintenues constantes au niveau de la situation de 19811986. qui est mise en relief dans la colonne de droite (encadrée) et qui aboutirait à un pourcentage de francophones après 35 ans de 84.5. Par exemple, si l'immigration quinquennale survivante résultante était maintenue à 216000 personnes durant 35 ans, les autres phénomènes étant maintenus à leur niveau de 1981-1986, le pourcentage des francophones après 35 ans passerait à $81,5 \%$. C'est la raison pour laquelle 216 est placé sur la figure au dessus de $81.5 \%$. Un autre exemple de l'interprètation de la figure se trouve dans l'illustration d'une modification de deux ou trois phénomènes. Si l'immigration se maintient comme dans l'exemple précédent à 216000 et si l'émigration se retrouve à 141000 durant toute la période, la figure montre que les francophones se retrouveront à environ $80,5 \%$ de la population québécoise, en diminution de $2 \%$ comme on peut le lire sur l'échelle inférieure.

\section{FIGURE 1 - SCÉNARIOS D'ÉVOLUTION DU POURCENTAGE DES FRANCOPHONES (LANGUE D'USAGE) DU QUEEBEC, À PARTIR DE LA SITUATION DE 1981}


quelle est la variation de chacun des phénomènes qui entraine une diminution ou une augmentation de $1 \%$ du pourcentage des francophones sur une période de 35 ans par comparaison à 1981. Par exemple, d'après les hypothèses du modèle mentionnées plus haut, une immigration quinquennale de 161000 personnes, toutes choses étant égales par ailleurs, entraîne une augmentation du groupe francophone de $1 \%$ sur 35 ans, tandis qu'une immigration de 216000 provoquerait une diminution de $1 \%$.

Le maintien constant sur 35 ans de tous les phénomènes à leur niveau de 1981-1986 entraînant une augmentation des francophones de $1,85 \%$ (de 82,55 à 84,40 ), nous avons également présenté ce résultat dans une colonne encadrée. De plus, certains phẻnomènes ne peuvent causer par leur seule variation des augmentations du groupe francophone de l'ordre de $1 \%$. C'est la raison pour laquelle nous avons présenté, à droite, des colonnes qui situent la variation du pourcentage des francophones qu'entraîneraient des variations extrêmes de ces phénomènes. Par exemple, si tous les transferts linguistiques des allophones sont dirigés vers l'anglais $10 \%$ vers les francophones sur la figure 1), l'augmentation du pourcentage des francophones est de $1,52 \%$ ce qui est supérieur au $1 \%$ que nous avons fixé comme marge supérieure de la plage de comparaison. De façon analogue, un indice synthétique de 1 enfant par femme (indice inférieur à tout ce qui a déjà été observê) permet une augmentation du pourcentage du groupe francophone de $1.55 \%$ sur 35 ans. Pour chacun de ces indices, nous avons également présenté une limite maximale.

\section{DISCUSSION}

Nous discuterons tout d'abord de l'impact des phénomènes sur le pourcentage des francophones au Québec pour parler ensuite des conséquences de ces variations pour leur effectif.

\section{Le pourcentage des francophones}

De ce qui précède, on voit qu'une augmentation quinquennale de l'ordre de 25000 immigrants (comprenant les entrants) maintenue constante sur toute la période, tous les autres phénomènes demeurant à leur niveau de 1981-1986, entraîne une diminution du poids relatif des francophones de l'ordre de $1 \%$ par rapport au niveau de 1981 (haut de la figure 1). Par 
exemple, alors qu'une immigration quinquennale de 139000 conduit au bout de 35 ans à un pourcentage de francophones de 84,5 , une immigration de 161000 seulement, toutes les autres hypothèses étant demeurées constantes, donne $83,5 \%$ après 35 ans, une diminution de $1 \%$ pour une augmentation quinquennale de 22000 de l'immigration résultante survivante. Une variation dans le sens opposé, du même ordre de grandeur, entraîne évidemment l'effet inverse. Il n'est donc pas surprenant que l'émigration ait l'effet opposé, entraînant une variation en sens contraire de $1 \%$ sur 35 ans pour chaque fluctuation de l'émigration de 25000 personnes.

De façon analogue, une diminution d'environ $6 \%$ de la proportion des francophones parmi les immigrants entraîne une diminution de $1 \%$ des francophones sur une même période (par exemple, de $34 \%$ à $27 \%$ ou de $27 \%$ à $21 \%$ ).

Ce qui précède vaut pour des changements d'une seule composante. Si, par exemple, on en fait varier deux, les effets se composent. Ainsi une augmentation d'un peu plus de 75000 immigrants alliée à une diminution de 25000 émigrants a pour consêquence une diminution du pourcentage des francophones de l'ordre de $4 \%$ par rapport à la norme 1981-1986 (+1,85\%) après 35 ans $(-1,88 \%$ si l'immigration est de 216000 et l'émigration de 141000 ).

La situation observée durant la période 1986-1991, bien qu'imparfaitement connue, semble correspondre à peu près à cette dernière hypothèse, mais le pourcentage des francophones parmi les immigrants a diminué et serait plutôt de d'ordre de $27 \%$ à $34 \%$. Une telle hypothèse entraînerait sur 35 ans une diminution des francophones de l'ordre de $-3,0 \%$ à $-4,5 \%$ par rapport au pourcentage observé en 1981 , le ramenant à un pourcentage moyen de 78,7 . Un tel résultat montre la sensibilité de l'équilibre linguistique à la migration. Il souligne indirectement l'impact relativement faible de la mobilité linguistique évoquée plus haut puisque même une mobilité linguistique des allophones favorable à $100 \%$ aux francophones donnerait environ $1 \%$ de plus aux francophones qui se retrouveraient à un peu plus de $79,7 \%$ (au bas de la figure) au lieu de 78,7 comme précédemment. 


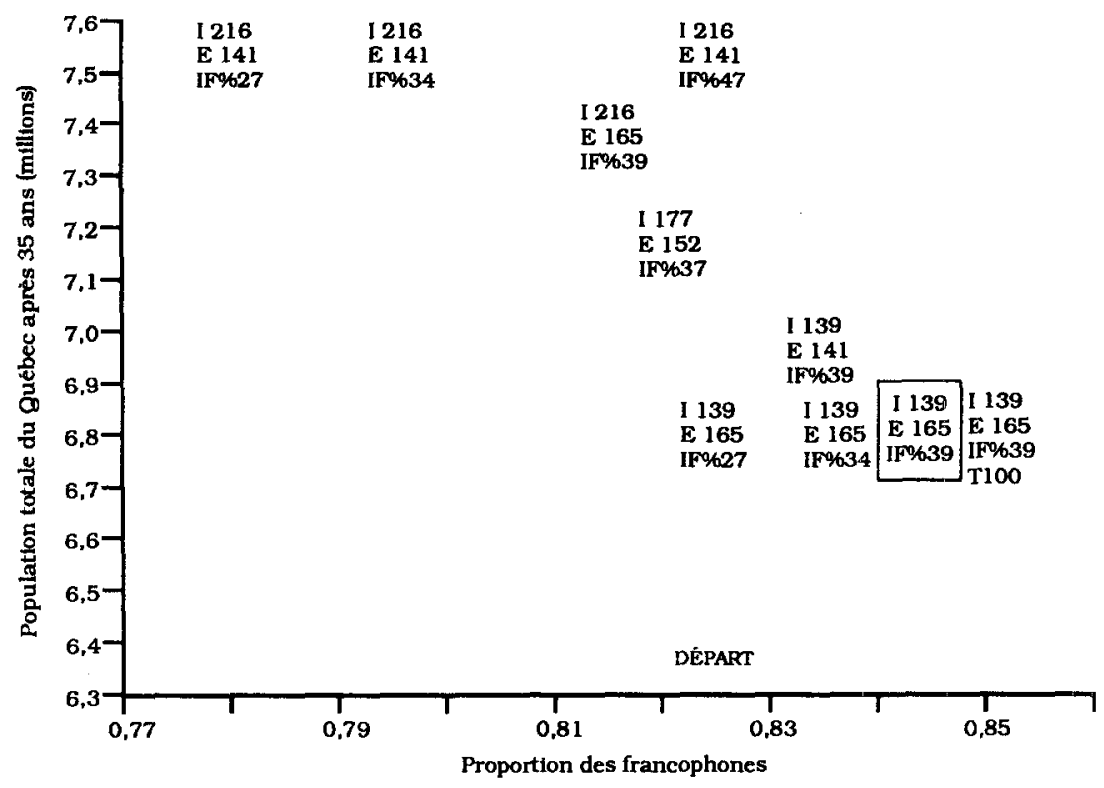

1 : Nombre d'immigrants survivants quinquennaux résultants.

$\mathrm{E}$ : Nombre d'émigrants survivants quinquennaux résultants.

IF\% : Pourcentage des immigrants francophones parmi les immigrants.

T100 : Selon cette hypothèse, toute la mobilité linguistique des allophones se fait vers le français.

NOTE - Par rapport à la figure 1. cette figure ajoute la dimension effectif en nombres absolus, qui se trouve en ordonnée. L'encadré correspond à la situation de 1981-1986.

\section{FIGURE 2 - POPULATION TOTALE DU QUEBEC APRES 35 ANS ET PROPORTION DE FRANCOPHONES (LANGUE DUUSAGE) EN FONCTION D'HYPOTHESES DE MIGRATION}

\section{L'effectif des francophones}

Nous avons discutê jusqu'ici de l'impact des phénomènes sur la proportion des francophones. On peut se demander également quel serait l'impact des différents scénarios sur l'effectif total de la population du Québec. C'est ce que présente la figure 2, qui met en relation le pourcentage des francophones et la population totale du Québec après 35 ans qui correspondent à chaque scénario. Chacun de ces scénarios maintient la fécondité, la mortalité et les transferts linguistiques (sauf mention contraire) à leur niveau estimé pour la période 1981-1986. L'immigration, le pourcentage des immigrants francophones ainsi que l'émigration sont maintenus constants 
au niveau indiqué sur la figure. Ainsi, par exemple, une êmigration quinquennale de 165000 personnes et une immigration de 139000 personnes dont $39 \%$ de francophones (I 139, E 165, IF\% 39) aboutissent après 35 ans à un pourcentage de francophones de 84,4 et à une population de 6806000 . Par contre, les hypothèses correspondant davantage à la situation de 1986-1991 (I 216, E 141, IF\% 27 ou 34), qui aboutissent à une diminution du pourcentage des francophones, correspondent à un accroissement de la population, celle-ci atteignant 7500000 après 35 ans.

Cette figure souligne un choix démographique auquel se voit confronté le Québec : la croissance de l'effectif de la population totale ou bien celle du pourcentage des francophones.

Une avenue qui pourrait sembler raisonnable consisterait à vouloir préserver la proportion des francophones à son niveau de départ. Un tel objectif laisse place à des choix touchant surtout l'immigration, puisque le contrôle de l'émigration demeure en général un vœu pieux, et que le contrôle des transferts linguistiques a un impact très limité.

Nous avons indiqué sur la figure 2 , au dessus du point de départ, quelques cheminements qui aboutissent après 35 ans à un maintien du pourcentage des francophones. Pour conserver l'immigration et l'émigration à leur niveau de 1986-1991, ll faudrait que $47 \%$ des immigrants soient francophones ${ }^{4}$ (I 216 , E 141, IF\% 47 au haut de la figure 2). La population du Guébec atteindrait alors plus de 7500000 au bout de 35 ans. Par contre si on conserve le niveau observé durant la période 1981-1986, le pourcentage d'immigrants francophones nécessaire au maintien de la proportion des francophones n'est que de $27 \%$ mais la population après 35 ans n'est que de 6800000 (I 139 . E 165, IF\% 27).

Une infinité de combinaisons de niveaux d'immigration, d'émigration, de pourcentage d'immigrants francophones et de transferts linguistiques peuvent permettre le maintien de la proportion des francophones, mais il ne faut pas se faire d'illusion sur l'impact de la mobilité linguistique, qui est infèrieur à $1 \%$. Il ne s'agit pas là d'un jugement sur l'importance

4 Il s'agit là d'un minimum, puisque notre modèle accorde aux immigrants installés au Québec la mobilité géographique de leur groupe linguistique, ce qui est sans doute un peu faible dans le cas des immigrants francophones, pour qui les dernières études semblent montrer une propension très forte à quitter le Québec. 
des lois linguistiques, mais d'une appréciation de leur impact démolinguistique global. Rappelons de plus que l'analyse que nous faisons pour l'ensemble du Québec prendrait un visage différent si nous l'appliquions à certaines régions.

\section{CONCLUSION}

Les politiques de population concernant les groupes linguistiques ne peuvent guère agir directement que sur l'immigration et, dans une moindre mesure, sur la mobilité linguistique. On verrait mal en effet une politique favorisant la fécondité d'un groupe, et toute action discriminatoire concernant la mortalité est impensable. Comme l'émigration ne se contrôle pas, il reste essentiellement, comme moyen de contrôle de l'équilibre entre les groupes linguistiques, l'immigration, sur laquelle on peut agir en modifiant son intensité et sa composition linguistique. En dépit de l'imprécision des clonnées disponibles en mars 1992, il semble que le maintien de la migration à son niveau de 1986-1991 aboutit après 35 ans à une diminution de la proportion des francophones. Cette diminution serait plus forte si le gouvernement appliquait sa politique d'immigration, qui prévoit une augmentation de l'immigration par rapport à la période récente allant jusqu'à 55000 immigrants par année dès 1994 (MCCI, 1990). Que des considérations humanitaires, économiques ou autres puissent justifier les politiques migratoires les plus diverses peut se comprendre, mais il est important à notre avis d'en voir les conséquences démolinguistiques. Il ne faut pas par ailleurs se faire trop d'illusions sur l'effet compensatoire de mesures concernant les autres phénomènes. Nous croyons qu'une meilleure compréhension de la dynamique des groupes linguistiques permettra l'action la plus rationnelle possible dans un domaine où l'émotivité n'est pas bonne conseillère.

\section{RÉFÉRENCES BIBLIOGRAPHIGUES}

BOURBEAU, R., 1986. Utilisation des données des recensements canadiens pour l'étude de la migration des groupes linguistiques au Québec, 1971, 1976 et 1981. Montréal, Université de Montréal, Département de démographie, rapport de recherche, $178 \mathrm{p}$.

CALDWELL, G., 1984. Out-Migration of 1971 English Mother-Tongue High School Leavers from Quebec: Eleven Years after. Lennoxville, Institut québécois de recherche sur la culture. 
CHARBONNEAU, H., J. HENRIPIN et J. LÉGARÉ, 1970. «L'avenir démographique des francophones au Québec et à Montréal en l'absence de politiques adéquates:, Revue de géographie de Montréal, XXIV, 2 : 199-202.

CHARBONNEAU, H., et R. MAHEU, 1973. Les Aspects démographiques de la question linguistique. Synthèse S3 réalisée pour le compte de la Commission d'enquête sur la situation de la langue française et sur les droits linguistiques au Québec, Québec, Éditeur officiel, $440 \mathrm{p}$.

GIRARD, C., 1992. Développement d'un modèle de projection et analyse de la sensibilité de la composition linguistique de la population du Québec. Montréal, Université de Montréal, Département de démographie, mémoire de maîtrise, $93 \mathrm{p}$. et annexes.

HENRIPIN, J., 1984. La Population québécoise de langue anglaise: une projection démolinguistique. Montréal, Alliance Québec, 21 p.

LACHAPELLE, R., et J. HENRIPIN, 1980. La Situation démolinguistique au Canada. Évolution passée et prospective. Montréal, L'Institut de recherches politiques, $391 \mathrm{p}$.

MCCI (Ministère des Communautés culturelles et de l'Immigration), 1990. Au Québec pour bâtir ensemble. Énoncé de politique en matière d'immigration et d'intégration. Québec, MCCI. Direction des communications, $88 \mathrm{p}$.

ROBITAILLE, N., et R. BOURBEAU, 1982. Perspectives de la population du Québec et de ses régions selon le groupe linguistique, 1971-2001. Étude préparée pour le Conseil de la langue française, Université de Montréal, Département de démographie, $86 \mathrm{p}$. et annexes.

TERMO'TE, M., et D. GAUVREAU, 1988. La Situation démolinguistique du Québec. Québec, Conseil de la langue française, 291 p. 


\section{RESUMÉ - SUMMARY - RESUMEN}

ROBITAILLE Norbert, BOURBEAU Robert, GIRARD Chantal et TREMBLAY Marc - LAVENIR DES GROUPES LINGUISTIQUES AU QUÉBEC : EGUILIBRES ET OPTIONS

Durant la période 1981-1986, l'équilibre des phénomènes démographiques au Québec était tel que la proportion de francophones avait tendance à s'accroitre. Cet article projette cette situation. ainsi que d'autres scénarios, et montre l'impact d'un accroissement de l'immigration sur le pourcentage de francophones au Buébec. It souligne, pour le Québec, l'antinomie qui existe entre la croissance de l'effectif de la population dans son ensemble et celle du pourcentage de francophones. Plus spécifiquement, il montre que la politique du gouvernement qui prévoit une immigration de 55000 immigrants annuellement entrainerait selon toute vraisemblance une diminution du pourcentage de francophones.

ROBITAILLE Norbert, BOURBEAU Robert, GIRARD Chantal and TREMBLAY Marc - THE FUTURE OF LINGUISTIC GROUPS IN QUEBEC: THE IMPACT OF IMMIGRATION

During the 1981-1986 period, the balance between demographic phenomena was such that the proportion of francophones in the population tended to increase in Quebec. In this article, we project the 1981-1986 situation and other scenarios to see the impact of increasing immigration on the proportion of francophones in Quebec. The results suggest that there is an antinomy between an increase of the total population and an increase of the proportion of francophones. Thus, the policy of the provincial government to accept 55000 immigrants per year would probably lead to a decrease in the proportion of francophones in Quebec.

ROBITAILLE Norbert, BOURBEAU Robert, GIRARD Chantal y TREMBLAY Marc - EL PORVENIR DE LOS GRUPOS LINGÜISTICOS EN GUÉBEC: EQUILIBRIOS Y OPCIONES

Durante el periodo 1981-1986, era tal el equilibrio de los fenómenos demográficos en Québec que la proporción de francófonos tendia a incrementarse. El presente articulo proyecta dicha situación, asi como otros aspectos, y demuestra el impacto que tiene el incremento de la inmigración sobre el porcentaje de francófonos en Québec. Subraya la antinomia que existe en Québec entre el crecimiento de la población en conjunto y el aumento del porcentaje de francófonos. Más específicamente, se demuestra que la política del gobierno, que prevé la entrada de 55000 inmigrantes al año, causaria muy probablemente una reducción del porcentaje de francófonos. 